\title{
Generalized Jacobi-Weierstrass operators and Jacobi expansions
}

\author{
José A. Adell ${ }^{1 *}$, Jorge Bustamante ${ }^{2 \dagger}$, Juan J. Merino ${ }^{3 \dagger}$ and José M. Quesada ${ }^{3 \dagger}$
}

\footnotetext{
"Correspondence: adell@unizar.es

'Departamento de Métodos

Estadísticos, Facultad de Ciencias,

Universidad de Zaragoza, Zaragoza,

Spain

Full list of author information is

available at the end of the article

${ }^{\dagger}$ Equal contributors
}

\begin{abstract}
We present a realization for some $K$-functionals associated with Jacobi expansions in terms of generalized Jacobi-Weierstrass operators. Fractional powers of the operators as well as results concerning simultaneous approximation and Nikolskii-Stechkin type inequalities are also considered.
\end{abstract}

MSC: 41A35; 47D06

Keywords: Jacobi-Weierstrass operators; Abel-Cartwright means; Realization of K-functionals; Semi-groups of operators; Fractional powers; Nikolskii-Stechkin type inequality

\section{Introduction}

In this note, we work with two fixed real parameters $\alpha$ and $\beta$ satisfying $\alpha \geq \beta \geq-1 / 2$. We use the following notations:

$$
\varrho^{\alpha, \beta}(x)=(1-x)^{\alpha}(1+x)^{\beta}, \quad x \in(-1,1),
$$

and, for $1 \leq p<\infty$,

$$
L_{(\alpha, \beta)}^{p}=\left\{f:[-1,1] \rightarrow \mathbb{R}:\|f\|_{p}=\left(\int_{-1}^{1}|f(x)|^{p} \varrho^{\alpha, \beta}(x) d x\right)^{1 / p}<\infty\right\}
$$

Moreover, for each $n \in \mathbb{N}_{0}, \mathbb{P}_{n}$ is the family of all algebraic polynomials of degree not greater than $n$,

$$
w_{n}^{\alpha, \beta}=\frac{(2 n+\alpha+\beta+1) \Gamma(n+\alpha+\beta+1) \Gamma(n+\alpha+1)}{\Gamma(n+\beta+1) \Gamma(n+1)(\Gamma(\alpha+1))^{2}}
$$

( $\Gamma$ stands for the gamma function) and

$$
\lambda_{n}=n(n+\alpha+\beta+1)
$$

Since $\alpha$ and $\beta$ are fixed, we set $X$ for one of the spaces $C[-1,1]$ or $L_{(\alpha, \beta)}^{p}$.

\section{Springer}


For $n \in \mathbb{N}$, the Jacobi polynomial $R_{n}^{(\alpha, \beta)}$ is the unique polynomial of degree $n$ which satisfies

$$
R_{n}^{(\alpha, \beta)}(1)=1 \quad \text { and } \quad \int_{-1}^{1} Q_{n-1}(x) R_{n}^{(\alpha, \beta)}(x) \varrho^{\alpha, \beta}(x) d x=0
$$

for all $Q_{n-1} \in \mathbb{P}_{n-1}$. We also take $R_{0}^{(\alpha, \beta)}(x)=1$.

For $f \in X$, the Fourier-Jacobi coefficients are defined by

$$
\left\langle f, R_{n}^{(\alpha, \beta)}\right\rangle=\int_{-1}^{1} f(x) R_{n}^{(\alpha, \beta)}(x) \varrho^{\alpha, \beta}(x) d x, \quad n \in \mathbb{N}_{0},
$$

and the associated expansion is

$$
f(x) \sim \sum_{n=0}^{\infty}\left\langle f, R_{n}^{(\alpha, \beta)}\right| w_{n}^{(\alpha, \beta)} R_{n}^{(\alpha, \beta)}(x)
$$

It is known that each $f \in L_{(\alpha, \beta)}^{1}$ is completely determined a.e. by its Fourier-Jacobi coefficients.

Definition 1.1 For fixed $\gamma>0$ and $t>0$, the generalized Jacobi-Weierstrass kernel is defined by

$$
W_{t, \gamma}(x)=\sum_{n=0}^{\infty} e^{-t \lambda_{n}^{\gamma}} w_{n}^{(\alpha, \beta)} R_{n}^{(\alpha, \beta)}(x), \quad x \in[-1,1]
$$

For $f \in X$, the generalized Jacobi-Weierstrass (or Abel-Cartwright) operator is defined by

$$
C_{t, \gamma}(f, x)=\int_{-1}^{1} \tau_{y}(f, x) W_{t, \gamma}(y) \varrho^{\alpha, \beta}(y) d y, \quad x \in[-1,1]
$$

where $\tau_{y}(f, x)$ is the translation given in Theorem 2.1 below.

Of course the kernel $W_{t, \gamma}$ and the operator $C_{t, \gamma}$ also depend on $\alpha$ and $\beta$ but, for simplicity, we omit these indexes. The (classical) Jacobi-Weierstrass operators correspond to $\gamma=1$.

The generalized Jacobi-Weierstrass operators have been studied in different papers, but only for parameters satisfying $0<\gamma \leq 1$. This restriction was considered because in such a case the kernels $W_{t, \gamma}$ are positive and the family $\left\{C_{t, \gamma}\right\}$ can be considered as formed by positive operators (see [2, 3], [7], pp. 96-97) and/or as a semigroup of contractions (see [2], pp. 49-52, and [18]). For $\gamma>1$, one cannot expect the positivity of $W_{t, \gamma}$. For instance, it is known that the analogous generalized Weierstrass kernels for trigonometric expansion are not positive when $\gamma>1$ (see [6], p. 263).

In this paper we will prove that the operators $C_{t, \gamma}$ can be used as a realization of some $K$-functionals which usually appear in some approximation problems related to Jacobi expansions. 
For fixed real $\gamma>0$, let $\Phi^{\gamma}(X)$ denote the family of all $f \in X$ for which there exists $\Psi^{\gamma}(f) \in$ $X$ satisfying

$$
\Psi^{\gamma}(f)(x) \sim \sum_{n=0}^{\infty} \lambda_{n}^{\gamma}\left\langle f, R_{n}^{(\alpha, \beta)}\right| w_{n}^{(\alpha, \beta)} R_{n}^{(\alpha, \beta)}(x)
$$

The associated $K$-functional is defined by

$$
K_{\gamma}(f, t)=K_{\gamma}(f, t)_{\alpha, \beta}=\inf _{g \in \Psi^{\gamma}(X)}\left\{\|f-g\|_{X}+t\left\|\Psi^{\gamma}(g)\right\|_{X}\right\}
$$

for $f \in X$ and $t>0$. For different realizations of these $K$-functionals, see [8], Theorem 7.1, and [10], Lemma 2.3. We will not use the characterization of these $K$-functionals in terms of moduli of smoothness. We will show that, for any $\gamma>0$,

$$
\sup _{0<s \leq t}\left\|\left(I-C_{s, \gamma}\right)(f)\right\|_{X} \approx K_{\gamma}(f, t)
$$

The notation $A(f, t) \approx B(f, t)$ means that there exists a positive constant $C$ such that $C^{-1} A(f, t) \leq B(f, t) \leq C A(f, t)$ with $C$ independent of $f$ and $t$.

Following [19], for $\gamma>0$, define

$$
\left(I-C_{t, 1}\right)^{\gamma}=\sum_{j=0}^{\infty}(-1)^{j}\left(\begin{array}{l}
\gamma \\
j
\end{array}\right) C_{j t, 1}
$$

where

$$
\left(\begin{array}{l}
\gamma \\
0
\end{array}\right)=1 \text { and }\left(\begin{array}{l}
\gamma \\
j
\end{array}\right)=\prod_{k=1}^{j} \frac{\gamma-k+1}{k} \quad \text { for } j \in \mathbb{N} \text {. }
$$

For these operators, we will show the relations

$$
K_{\gamma}\left(f, t^{\gamma}\right) \approx \sup _{0<s \leq t}\left\|\left(I-C_{s, 1}\right)^{\gamma}(f)\right\|_{X} \approx \sup _{0<s \leq t \gamma}\left\|\left(I-C_{s, \gamma}\right)(f)\right\|_{X}
$$

It is known that, if $Q_{n}$ is a trigonometric polynomial of degree not greater than $n$ and $r \in \mathbb{N}$, then

$$
\left\|Q_{n}^{(r)}\right\|_{p} \leq\left(\frac{n}{2 \sin (n h)}\right)^{r}\left\|\left(1-T_{h}\right)^{r}\left(Q_{n}\right)\right\|_{p}, \quad h \in(0, \pi / n),
$$

where $\|\cdot\|_{p}$ denotes the $L^{p}$-norm of $2 \pi$-periodic functions and $T_{h}$ is the translation operator. That is, $T_{h} Q(x)=Q(x+h)$. These inequalities are due to Nikolskii [11] and Stechkin [13]. For similar inequalities for algebraic polynomials, see [4] and the references given there. Here we will verify an analogous inequality by considering the operators $\Psi^{r}$ and the linear combination of the Jacobi-Weierstrass operators $C_{t, 1}$.

In Sect. 2 we collect some definitions and results which will be needed later. The main results are given in Sect. 3, where the result concerning simultaneous approximation is also included. Finally, in Sect. 4 we present a Nikolskii-Stechkin type inequality. 


\section{Auxiliary results}

We need a convolution structure due to Askey and Wainger (see [1]).

Theorem 2.1 For each $h \in[-1,1)$, there exists a function $\tau_{h}: X \rightarrow X$ with the following properties:

(i) For each $f \in X$, one has

$$
\left\|\tau_{h} f\right\|_{X} \leq\|f\|_{X}, \quad \lim _{h \rightarrow 1-}\left\|\tau_{h}(f)-f\right\|_{X}=0
$$

and

$$
\left\langle\tau_{h}(f), R_{n}^{(\alpha, \beta)}\right\rangle=R_{n}^{(\alpha, \beta)}(h)\left\langle f, R_{n}^{(\alpha, \beta)}\right\rangle, \quad n \in \mathbb{N}_{0} .
$$

(ii) For $f \in X$ and $g \in L_{\alpha, \beta}^{1}$, the integral

$$
(f * g)(x):=\int_{-1}^{1} \tau_{y}(f, x) g(y) \varrho^{\alpha, \beta}(y) d y
$$

exists a.e. in $[-1.1]$

$$
f * g=g * f, \quad f * g \in X, \quad\|f * g\|_{p} \leq\|g\|_{1}\|f\|_{X}
$$

and

$$
\left\langle f * g, R_{n}^{(\alpha, \beta)}\right\rangle=\left\langle f, R_{n}^{(\alpha, \beta)}\right\rangle\left\langle g, R_{n}^{(\alpha, \beta)}\right\rangle, \quad n \in \mathbb{N}_{0} .
$$

For $j>\alpha+1 / 2$ and $f \in X$, let

$$
S_{m}^{j}(f)=\sum_{k=0}^{m} \frac{A_{m-k}^{j}}{A_{m}^{j}}\left\langle f, R_{k}^{\alpha, \beta)}\right| w_{k}^{\alpha, \beta} R_{k}^{(\alpha, \beta)}(x), \quad A_{m}^{j}=\left(\begin{array}{c}
m+j \\
m
\end{array}\right)
$$

be the $m$ th Cesàro means of order $j$. It is known that there exists a constant $C$ such that

$$
\left\|S_{m}^{j}\right\| \leq C
$$

and, for each $f \in X$, one has ([2], Corollary 3.3.3, or [7], Theorem A)

$$
\lim _{m \rightarrow \infty}\left\|f-S_{m}^{j}(f)\right\|_{X}=0
$$

We need some classical results related to Banach spaces.

Definition 2.2 Let $Y$ be a real Banach space and $B(Y)$ be the Banach algebra of all bounded linear operators $B: Y \rightarrow Y$. A uniformly bounded family of operators $\{T(t)$ : $t \geq 0\}$ in $B(Y)$ is called an equi-bounded semigroup of class $\left(C_{0}\right)$ if

$$
T(s) T(t)=T(s+t) \quad \text { for } s, t \geq 0, \quad T(0)=I,
$$

and $\lim _{t \rightarrow 0+}\|f-T(t) f\|_{Y}=0$ for each $f \in Y$. 
Let $Y, B(Y)$ and $\{T(t): t>0\}$ be an equi-bounded semigroup as in Definition 2.2. Let $D(Q)$ be the family of all $g \in Y$, for which there exists $Q(g) \in Y$ such that

$$
Q(g)=\lim _{t \rightarrow 0+} \frac{1}{t}[T(t)-I] g
$$

(the limit is considered in the norm of $Y$ ). The operator $Q: D(Q) \rightarrow Y$ is called the infinitesimal generator of the semigroup $\{T(t): t \geq 0\}$. It is known that $Q$ is a closed linear operator and $D(Q)$ is dense in $Y$. For properties of semigroups of operators, see [5].

For $r \in \mathbb{N}$, set

$$
D\left(Q^{r+1}\right)=\left\{f \in Y: f \in D\left(Q^{r}\right) \text { and } Q^{r}(f) \in D(Q)\right\}
$$

and, for $f \in D\left(Q^{r+1}\right)$,

$$
Q^{r+1}(f)=Q\left(Q^{r}(f)\right)
$$

A family of operators $S=\left\{S_{t}, t>0\right\}, S_{t} \in B(Y)$ for each $t>0$ is called a (commutative) strong approximation process for $Y$ if, for all $f \in Y$ and $s, t>0$,

$$
S_{s}\left(S_{t}(f)\right)=S_{t}\left(S_{s}(f)\right), \quad\left\|S_{t}(f)\right\|_{Y} \leq \Lambda\|f\|_{Y} \quad \text { and } \quad \lim _{t \rightarrow 0+}\left\|f-S_{t}(f)\right\|_{Y}=0
$$

where $\Lambda$ is a constant. In such a case, we set

$$
\theta_{S}(f, t)=\sup _{0<s \leq t}\left\|f-S_{s}(f)\right\|_{Y} .
$$

Let $\phi:[0,1) \rightarrow \mathbb{R}^{+}$be a positive increasing function, $\phi(t) \rightarrow 0$ as $t \rightarrow 0$, and $Y_{0}$ be a subspace of $Y$. We say that $S$ is saturated with order $\phi$ and with trivial subspace $Y_{0}$ if every $f \in Y$ satisfying

$$
\lim _{t \rightarrow 0+} \frac{\theta_{S}(f, t)}{\phi(t)}=0
$$

belongs to $Y_{0}$ and there exists $f \in Y \backslash Y_{0}$ satisfying $\theta_{S}(f, t) \leq C(f) \phi(t)$. The following assertion is known (for instance, see [2], Theorem 2.4.2).

Theorem 2.3 Assume that $Y$ is a Banach space, $D(B)$ is a dense subspace of $Y$, and $B$ : $D(B) \rightarrow Y$ is a closed linear operator. Let $S=\left\{S_{t}: t>0\right\}$ be a strong approximation process in $Y$ satisfying $S_{t}(f) \in D(B)$ for any $f \in Y$ and each $t>0$. If there exists a constant $\gamma_{0}$ such that, for all $g \in D(B)$,

$$
\lim _{t \rightarrow 0+}\left\|\frac{S_{t}(g)-g}{t^{\gamma_{0}}}-B(g)\right\|_{Y}=0
$$

then the strong approximation process $S$ is saturated with order $t^{\gamma_{0}}$ and the trivial space is the kernel of $B$. 


\section{The operators $C_{t, y}$ as a semigroup}

In fact, it is known that, for $x \in(-1,1),\left|R_{n}^{(\alpha, \beta)}(x)\right|<1,[14]$, pp. 163-164, and there exists a constant $C$ such that, for each $n \in \mathbb{N}_{0}$,

$$
w_{n}^{(\alpha, \beta)} \leq C n^{2 \alpha+1} .
$$

These relations can be used to prove that the series in (5) converges absolutely and uniformly in $[-1,1]$. Thus $W_{t, \gamma} \in L_{(\alpha, \beta)}^{1}$ and, for each $f \in L_{(\alpha, \beta)}^{1}$, the series $C_{t, \gamma}(f)$ converges absolutely and uniformly in $[-1,1]$. Moreover,

$$
C_{t, \gamma}(f, x)=\left(W_{t, \gamma} * f\right)(x)=\sum_{n=0}^{\infty} e^{-t \lambda_{n}^{\gamma}}\left\langle f, R_{n}^{(\alpha, \beta)}\right\rangle w_{n}^{(\alpha, \beta)} R_{n}^{(\alpha, \beta)}(x) .
$$

For these assertions, see [2], p. 30.

Our first result seems to be known. For convenience of the reader, we include a proof.

Theorem 3.1 For each $\gamma>0$, the family of operators $\left\{C_{t, \gamma}: t>0\right\}$ is an equi-bounded semigroup of operators in $X$.

Proof. It follows from Theorem 3.9 of [15] that the family of operators $\left\{C_{t, \gamma}: t>0\right\}$ is uniformly bounded.

Condition (12) is derived from the properties of the convolution. In fact, it follows from (9) that, for each $f \in X$ and $k \in \mathbb{N}_{0}$,

$$
\begin{aligned}
\left\langle C_{s+t}(f), R_{k}^{(\alpha, \beta)}\right\rangle & =e^{-(s+t) \lambda_{n}^{\gamma}}\left\langle f, R_{k}^{(\alpha, \beta)}\right\rangle=e^{-s \lambda_{n}^{\gamma}}\left\langle C_{t, \gamma}(f), R_{k}^{(\alpha, \beta)}\right\rangle \\
& =\left\langle C_{s, \gamma}\left(C_{t, \gamma}(f)\right), R_{k}^{(\alpha, \beta)}\right\rangle
\end{aligned}
$$

and this implies $C_{s+t}(f)=\left(C_{s, \gamma} \circ C_{t, \gamma}\right)(f)$.

Finally, for each $k \in \mathbb{N}_{0}$,

$$
C_{t, \gamma}\left(R_{k}^{(\alpha, \beta)}\right)(x)=e^{-t \lambda_{n}^{\gamma}} R_{k}^{(\alpha, \beta)}(x)
$$

Hence

$$
\lim _{t \rightarrow 0+}\left\|R_{k}^{(\alpha, \beta)}-C_{t, \gamma}\left(R_{k}^{(\alpha, \beta)}\right)\right\|_{X}=0 .
$$

Since the operators $C_{t, \gamma}$ are linear and uniformly bounded and the polynomials are dense in $X$, the last equation holds for every $f \in X$.

Taking into account Theorem 3.1, we denote by $A_{\gamma}$ the infinitesimal generator of $C_{t, \gamma}$ and by $D\left(A_{\gamma}\right)=D\left(A_{\gamma}(\alpha, \beta)\right)$ the domain of $A_{\gamma}$. In the next result we give a description of the infinitesimal generator.

Theorem 3.2 If $\gamma, t>0$ and $A_{\gamma}: D\left(A_{\gamma}\right) \rightarrow X$ is the infinitesimal generator of $C_{t, \gamma}$, then

$$
D\left(A_{\gamma}\right)=\Psi^{\gamma}(X) \text { and }-A_{\gamma}(f)=\Psi^{\gamma}(f)
$$

for each $f \in \Psi^{\gamma}(X)$. 
Moreover, for each $r \in \mathbb{N}$ and $f \in D\left(A_{\gamma}^{r}\right)$,

$$
D\left(A_{\gamma}^{r}\right)=\Psi^{r \gamma}(X) \text { and }(-1)^{r} A_{\gamma}^{r}(f)=\Psi^{r \gamma}(f)
$$

where $A_{\gamma}^{r}$ is defined as in (14).

Proof Since $A_{\gamma}$ is the infinitesimal generator of the semi-group (see (13)), $A_{\gamma}: D\left(A_{\gamma}\right) \rightarrow X$ is a closed operator.

If $f \in D\left(A_{\gamma}\right)$, then

$$
\left\langle A_{\gamma}(f), R_{n}^{(\alpha, \beta)}\right\rangle=\lim _{t \rightarrow 0+} \frac{1}{t}\left(e^{-t \lambda_{n}^{\gamma}}-1\right)\left\langle f, R_{n}^{(\alpha, \beta)}\right\rangle=-\lambda_{n}^{\gamma}\left\langle f, R_{n}^{(\alpha, \beta)}\right\rangle
$$

Thus $f \in \Psi^{\gamma}(X)$ and

$$
\Psi^{\gamma}(f)=-A_{\gamma}(f)
$$

In particular, for each polynomial $P$, one has $P \in D\left(A_{\gamma}\right)$ and $\Psi^{\gamma}(P)=-A_{\gamma}(P)$.

On the other hand, fix an integer $j>\alpha+1 / 2$. For $f \in \Psi^{\gamma}(X)$, let $S_{m}^{j}(f)$ and $S_{m}^{j}\left(\Psi^{\gamma}(f)\right)$ be the $m$ th Cesàro means of order $j$ of $f$ and $\Psi^{\gamma}(f)$, respectively. We know that (see (11))

$$
S_{m}^{j}(f) \rightarrow f, \quad m \rightarrow \infty
$$

and

$$
-A_{\gamma}\left(S_{m}^{j}(f)\right)=\Psi^{\gamma}\left(S_{m}^{j}(f)\right)=S_{m}^{j}\left(\Psi^{\gamma}(f)\right) \rightarrow \Psi^{\gamma}(f)
$$

Since $-A_{\gamma}$ is a closed operator, $f \in D\left(A_{\gamma}\right)$ and $-A_{\gamma}(f)=\Psi^{\gamma}(f)$.

Equations (18) can be proved by recurrence. For instance, (19) can be written as

$$
\left\langle A_{\gamma}^{2}(f), R_{n}^{(\alpha, \beta)}\right\rangle=\left\langle A_{\gamma}\left(A_{\gamma}(f)\right), R_{n}^{(\alpha, \beta)}\right\rangle=-\lambda_{n}^{\gamma}\left\langle A_{\gamma}(f), R_{n}^{(\alpha, \beta)}\right\rangle=\lambda_{n}^{2 \gamma}\left\langle f, R_{n}^{(\alpha, \beta)}\right\rangle .
$$

Theorem 3.3 (i) If for $\gamma, t>0$, and $f \in X$

$$
\theta_{\gamma}(f, t)=\theta_{\gamma}(f, t)_{\alpha, \beta}=\sup _{0<s \leq t}\left\|\left(I-C_{s, \gamma}\right)(f)\right\|,
$$

and $K_{\gamma}(f, t)$ is defined by $(7)$, then

$$
\theta_{\gamma}(f, t) \approx K_{\gamma}(f, t)
$$

(ii) The strong approximation process $\left\{C_{t, \gamma} ; t>0\right\}$ is saturated with order $t$ and the trivial class consists of the constant functions.

Proof (i) From Theorem 3.2 we know that $-\Psi^{\gamma}$ is the infinitesimal generator of $\left\{C_{t, \gamma}\right\}$ and $D\left(A_{\gamma}\right)=\Psi^{\gamma}(X)$. Thus, the result is a simple consequence of [17], Theorem 1.1, or [5], p. 192. 
(ii) We will derive the result from Theorem 2.3, with $B=\Psi^{\gamma}$ and $D(B)=D\left(A_{\gamma}\right)$. We should verify that $C_{t, \gamma}(f) \in D\left(A_{\gamma}\right)$ for any $f \in X$ and each $t>0$.

For any $f \in X$, the Fourier-Jacobi coefficients of $f$ are bounded by $\|f\|_{L_{(\alpha, \beta)}^{1}}$. Taking into account (16), for every $x \in[-1,1]$,

$$
\begin{aligned}
& \mid \sum_{n=1}^{\infty} \lambda_{n}^{\gamma} \exp \left\{-t \lambda_{n}\right\}\left\{f, R_{n}^{(\alpha, \beta)}\left|w_{n}^{(\alpha, \beta)} R_{n}^{(\alpha, \beta)}(x)\right|\right. \\
& \quad \leq\|f\|_{L_{(\alpha, \beta)}^{1}} \sum_{n=1}^{\infty} \lambda_{n}^{\gamma} \exp \left\{-t \lambda_{n}^{\gamma}\right\} w_{n}^{(\alpha, \beta)} \\
& \quad \leq C\|f\|_{L_{(\alpha, \beta)}^{1}} \sum_{n=1}^{\infty} \lambda_{n}^{\gamma} \exp \left\{-t \lambda_{n}^{\gamma}\right\} n^{2 \alpha+1}<\infty .
\end{aligned}
$$

Since the series converges absolutely and uniformly, it defines a function $g_{t} \in X$ satisfying

$$
\left\langle g_{t}, R_{n}^{(\alpha, \beta)}\right\rangle=\lambda_{n}^{\gamma} \exp \left\{-t \lambda_{n}^{\gamma}\right\}\left\langle f, R_{n}^{(\alpha, \beta)}\right\rangle=\lambda_{n}^{\gamma}\left\langle C_{t, \gamma}(f), R_{n}^{(\alpha, \beta)}\right\rangle, \quad n \in \mathbb{N}
$$

By definition of the operator $\Psi^{\gamma}, C_{t, \gamma}(f) \in \Psi^{\gamma}(X)$ (Theorem 3.2) and

$$
\Psi^{\gamma}\left(C_{t, \gamma}(f)\right)=g_{t}
$$

We have proved that $C_{t, \gamma}(X) \in D\left(A_{\gamma}\right)$.

If $g \in \Psi^{\gamma}(X)=D\left(A_{\gamma}\right)$, by definition of the infinitesimal generator,

$$
\lim _{t \rightarrow 0+}\left\|\frac{C_{t, \gamma}(g)-g}{t}-A_{\gamma}(g)\right\|_{Y}=0
$$

If $f \in \Psi^{\gamma}(X)$ and $A_{\gamma}(f)=-\Psi^{\gamma}(f)=0$, then $\left\langle f, R_{n}^{(\alpha, \beta)}\right\rangle=0$ for all $n \in \mathbb{N}$. Therefore $f$ is a constant.

From part (i), if $g \in \Psi^{\gamma}(X)$, then

$$
\theta_{\gamma}(g, t) \leq C K_{\gamma}(g, t) \leq C t\left\|\Psi^{\gamma}(g)\right\|_{X}
$$

Hence, the family

$$
\left\{f \in X: \exists C(f) \text { such that } \theta_{\gamma}(f, t) \leq C(f) t\right\}
$$

contains nonconstant functions.

Now, from Theorem 2.3, we know that the strong approximation process $\left\{C_{t, \gamma}: t>0\right\}$ is saturated with order $t$.

Remark 3.4 Some characterizations of the saturation class of the strong approximation process $\left\{C_{t, \gamma}: t>0\right\}$ can be given as in [2], Theorems 5.1.1 and 7.4.1, where the case $\gamma=1$ was considered. When $\gamma>0$ is not an integer, fractional derivatives should be considered. This task would lead us far from our main topic. 
Remark 3.5 A relation similar to (i) in Theorem 3.3 is asserted in [16], p. 2885, for the discrete case and Gauss-Weierstrass type means

$$
\widetilde{W}_{\Omega(n), \gamma}(f, x)=\sum_{n=0}^{\infty} e^{-(\Omega(k) / \Omega(n))^{\gamma}}\left\langle f, R_{n}^{(\alpha, \beta)}\right| w_{n}^{(\alpha, \beta)} R_{n}^{(\alpha, \beta)}(x),
$$

with $\Omega$ varying in a specified class of functions. The proof suggested there is different from the one given here (it does not use the semi-group structure). The main argument in [16] is that some abstract Riesz means are equivalent (as approximation processes) to some Gauss-Weierstrass type means. This kind of equivalence can also be derived by using Corollary 5.4 of [9]. Anyway, the arguments of [16] and the proof given here are related because both use [15], Theorem 3.9, to obtain a uniformly bounded family of multipliers. Apart from this, other topics considered here are not connected with [16].

The arguments used in the proof of Theorem 3.2 can be used to derive similar relations concerning the fractional powers of the Jacobi-Weierstrass operators $\left\{C_{t, 1}\right\}$.

Recall that $A_{1}: D\left(A_{1}\right) \rightarrow X$ is the infinitesimal generator of $\left\{C_{t, 1}, t>0\right\}$. For $\gamma>0$, let $D\left(\left(-A_{1}\right)^{\gamma}, X\right)$ be the family of all $f \in X$, for which there exists an element $\left(-A_{1}\right)^{\gamma}(f) \in X$ satisfying

$$
\lim _{t \rightarrow 0+}\left\|\left(-A_{1}\right)^{\gamma}(f)-\frac{1}{t^{\gamma}}\left(I-C_{t, 1}\right)^{\gamma}(f)\right\|_{X}=0,
$$

where $\left(I-C_{t, 1}\right)^{\gamma}(f)$ is defined by (8). This induces a map

$$
\left(-A^{1}\right)^{\gamma}: D\left(\left(-A^{1}\right)^{\gamma}, X\right) \rightarrow X
$$

which is called the fractional power of order $\gamma$ of $-A_{1}$.

Proposition 3.6 If $\gamma>0$ and $\left(-A_{1}\right)^{\gamma}$ is the fractional power of order $\gamma$ of $-A_{1}$, then

$$
D\left(\left(-A_{1}\right)^{\gamma}, X\right)=\Psi^{\gamma}(X)
$$

and, for each $f \in \Psi^{\gamma}(X)$,

$$
\Psi^{\gamma}(f)=\lim _{t \rightarrow 0+} \frac{1}{t^{\gamma}}\left(I-C_{t, 1}\right)^{\gamma}(f)=\lim _{t \rightarrow 0+} \frac{1}{t}\left(f-C_{t, \gamma}(f)\right) .
$$

Proof If $\gamma$ is a positive integer or $|a|<1$, the Taylor expansion gives

$$
(1-a)^{\gamma}=\sum_{j=0}^{\infty}(-1)^{j}\left(\begin{array}{l}
\gamma \\
j
\end{array}\right) a^{j}
$$

Notice that

$$
\left\langle\left(I-C_{t, 1}\right)^{\gamma}(f), R_{n}^{(\alpha, \beta)}\right\rangle=\sum_{k=0}^{\infty}(-1)^{k}\left(\begin{array}{l}
\gamma \\
k
\end{array}\right)\left\langle C_{k t, 1}(f), R_{n}^{(\alpha, \beta)}\right\rangle
$$




$$
\begin{aligned}
& \left.=\sum_{k=0}^{\infty}(-1)^{k}\left(\begin{array}{l}
\gamma \\
k
\end{array}\right)\left\langle W_{k t}, R_{n}^{(\alpha, \beta)}\right\rangle f f, R_{n}^{(\alpha, \beta)}\right\rangle \\
& \left.=\left\langle f, R_{n}^{(\alpha, \beta)}\right\rangle \sum_{k=0}^{\infty}(-1)^{k}\left(\begin{array}{l}
\gamma \\
k
\end{array}\right) \exp \left(-k t \lambda_{n}\right)\right) \\
& =\left\langle f, R_{n}^{(\alpha, \beta)}\right\rangle\left(1-\exp \left(-t \lambda_{n}\right)\right)^{\gamma} .
\end{aligned}
$$

Therefore, if $f \in D\left(\left(-A_{1}\right)^{\gamma}, X\right)$, then

$$
\left\langle\left(-A_{1}\right)^{\gamma}(f), R_{n}^{(\alpha, \beta)}\right\rangle=\left(\lambda_{n}\right)^{\gamma}\left\langle f, R_{n}^{(\alpha, \beta)}\right\rangle .
$$

Hence $f \in \Psi^{\gamma}(X)$ and $\left(-A_{1}\right)^{\gamma}(f)=\Psi^{\gamma}(f)$.

It is clear that, for each polynomial $P$, one has $P \in D\left(\left(-A_{1}\right)^{\gamma}, X\right)$ and

$$
\left(-A_{1}\right)^{\gamma}(P)=\Psi^{\gamma}(P)
$$

On the other hand, fix an integer $j>\alpha+1 / 2$. For $f \in \Psi^{\gamma}(X)$, let $S_{m}^{j}(f)$ and $S_{m}^{j}\left(\Psi^{\gamma}(f)\right)$ be the $m$ th Cesàro means of order $j$ of $f$ and $\Psi^{\gamma}(f)$, respectively. From (11), as in the proof of Theorem 3.2, one has $\lim _{m \rightarrow \infty}\left\|S_{m}^{j}(f)-f\right\|_{X}=0$ and

$$
\begin{aligned}
\lim _{m \rightarrow \infty}\left\|\left(-A^{1}\right)^{\gamma}\left(S_{m}^{j}(f)\right)-\Psi^{\gamma}(f)\right\|_{X} & =\lim _{m \rightarrow \infty}\left\|\Psi^{\gamma}\left(S_{m}^{j}(f)\right)-\Psi^{\gamma}(f)\right\|_{X} \\
& =\lim _{m \rightarrow \infty}\left\|S_{m}^{j}\left(\Psi^{\gamma}(f)\right)-\Psi^{\gamma}(f)\right\|_{X}=0 .
\end{aligned}
$$

It was proved in [19], Theorem 4, that $D\left(\left(-A_{1}\right)^{\gamma}, X\right)$ is dense in $X$ and $\left(-A_{1}\right)^{\gamma}$ is a closed operator. Hence $f \in D\left(\left(-A_{1}\right)^{\gamma}, X\right)$ and $\left(-A_{1}\right)^{\gamma}(f)=\Psi^{\gamma}(f)$.

The last equality in (21) was proved in Theorem 3.2, because $\Psi^{\gamma}$ is the infinitesimal generator of $\left\{C_{t, \gamma}, t>0\right\}$.

Theorem 3.7 For fixed $\gamma>0$, one has

$$
K_{\gamma}\left(f, t^{\gamma}\right) \approx \sup _{0<s \leq t}\left\|\left(I-C_{s, 1}\right)^{\gamma}(f)\right\|_{X} \approx \theta_{\gamma}\left(f, t^{\gamma}\right)
$$

for each $f \in X$ and $t>0$.

Proof From Theorems 3.1 and 3.2 we know that the family $\left\{C_{t, 1}, t \geq 0\right\}$ is a semi-group of operators of class $\left(C_{0}\right)$ with the infinitesimal generator $A_{1}=-\Psi^{1}$. From Theorem 1.1 of [17], we know that, for all $f \in X$ and $t>0$,

$$
\inf _{g \in D\left(\left(-A_{1}\right)^{\gamma}, X\right)}\left(\|f-g\|_{X}+t^{\gamma}\left\|\left(-A_{1}\right)^{\gamma}(g)\right\|_{X}\right) \approx \sup _{0<s \leq t}\left\|\left(I-C_{s, 1}\right)^{\gamma}(f)\right\|_{X}
$$

where $\left(-A_{1}\right)^{\gamma}$ is given as in (20). But it was verified in Proposition 3.6 that $\Psi^{\gamma}(X)=$ $D\left(\left(-A_{1}\right)^{\gamma}, X\right)$ and $\left(-A_{1}\right)^{\gamma}(g)=\Psi^{\gamma}(g)$ for each $g \in \Psi^{\gamma}(X)$.

The equivalence with $\theta_{\gamma}\left(f, t^{\gamma}\right)$ follows from Theorem 3.3.

Remark 3.8 When $\gamma$ is an integer, Theorem 3.7 is similar to the Main Theorem in [18], p. 390, but the authors assumed that the operators are positive (plus other conditions). 
Remark 3.9 The results of Theorem 3.7 allow us to obtain equivalent relations between fractional powers $\left(I-C_{s, 1}\right)^{\gamma}$ and some Riesz means as in Theorem 5.1 of [9].

Some result concerning simultaneous approximation can be derived from the ones given above.

Theorem 3.10 If $\gamma, \sigma$, and $t$ are positive real numbers and $f \in \Psi^{\sigma}(X)$, then

$$
\begin{aligned}
& C_{t, \gamma}(f),\left(I-C_{t, 1}\right)^{\gamma}(f) \in \Psi^{\sigma}(X), \\
& \left\|\Psi^{\sigma}(f)-\Psi^{\sigma}\left(C_{t, \gamma}(f)\right)\right\|_{X} \leq C \theta_{\gamma}\left(\Psi^{\sigma}(f), t\right)
\end{aligned}
$$

and

$$
\left\|\Psi^{\sigma}\left(\left(I-C_{t, 1}\right)^{\gamma}(f)\right)\right\|_{X} \leq C \theta_{\gamma}\left(\Psi^{\sigma}(f), t^{\gamma}\right),
$$

where the constant $C$ is independent of $f$ and $t$.

Proof If $f \in \Psi^{\sigma}(X)$ and $n \in \mathbb{N}_{0}$, from (17) we obtain

$$
\begin{aligned}
\left\langle C_{t, \gamma}\left(\Psi^{\sigma}(f)\right), R_{n}^{(\alpha, \beta)}\right\rangle & =\exp \left(-t \lambda_{n}^{\gamma}\right)\left\langle\Psi^{\sigma}(f), R_{n}^{(\alpha, \beta)}\right\rangle \\
& =\lambda_{n}^{\sigma} \exp \left(-t \lambda_{n}^{\gamma}\right)\left\langle f, R_{n}^{(\alpha, \beta)}\right\rangle=\lambda_{n}^{\sigma}\left\langle C_{t, \gamma}(f), R_{n}^{(\alpha, \beta)}\right\rangle
\end{aligned}
$$

and from (22) one has

$$
\begin{aligned}
\left\langle\left(I-C_{t, 1}\right)^{\gamma}\left(\Psi^{\sigma}(f)\right), R_{n}^{(\alpha, \beta)}\right\rangle & =\left(1-\exp \left(-t \lambda_{n}\right)\right)^{\gamma}\left\langle\Psi^{\sigma}(f) ; R_{n}^{(\alpha, \beta)}\right\rangle \\
& =\lambda_{n}^{\sigma}\left(1-\exp \left(-t \lambda_{n}\right)\right)^{\gamma}\left\langle f, R_{n}^{(\alpha, \beta)}\right\rangle=\lambda_{n}^{\sigma}\left\langle\left(I-C_{t, 1}\right)^{\gamma}(f), R_{n}^{(\alpha, \beta)}\right\rangle
\end{aligned}
$$

Therefore $C_{t, \gamma}(f),\left(I-C_{t, 1}\right)^{\gamma}(f) \in \Psi^{\sigma}(X)$,

$$
\Psi^{\sigma}\left(C_{t, \gamma}(f)\right)=C_{t, \gamma}\left(\Psi^{\sigma}(f)\right) \quad \text { and } \quad \Psi^{\sigma}\left(\left(I-C_{t, 1}\right)^{\gamma}(f)\right)=\left(I-C_{t, 1}\right)^{\gamma}\left(\Psi^{\sigma}(f)\right)
$$

Now, from Theorem 3.3 one has

$$
\left\|\Psi^{\sigma}(f)-\Psi^{\sigma}\left(C_{t, \gamma}\right)\right\|_{X}=\left\|\left(I-C_{t, \gamma}\right)\left(\Psi^{\sigma}(f)\right)\right\|_{X} \leq C \theta_{\gamma}\left(\Psi^{\sigma}(f), t\right),
$$

and using Theorem 3.7 we obtain

$$
\left\|\Psi^{\sigma}\left(\left(I-C_{t, 1}\right)^{\gamma}(f)\right)\right\|_{X}=\left\|\left(I-C_{t, 1}\right)^{\gamma}\left(\Psi^{\sigma}(f)\right)\right\|_{X} \leq C \theta_{\gamma}\left(\Psi^{\sigma}(f), t^{\gamma}\right) .
$$

\section{A Nikolskii-Stechkin type inequality}

Theorem 4.1 For each $r \in \mathbb{N}$, there exists a constant $C$, depending upon $r$, such that, for every $\lambda \geq 1$ and for each polynomial $P \in \mathbb{P}_{\xi(\lambda)}$,

$$
\left\|\Psi^{r}(P)\right\|_{X} \leq C \lambda^{r} \sup _{0<h \leq 1 / \lambda}\left\|\left(I-C_{h, 1}\right)^{r}(P)\right\|_{X}
$$


where

$$
\xi(\lambda)=\max \left\{k \in \mathbb{N}_{0}: k(k+\alpha+\beta+1)<\lambda\right\} .
$$

Proof In this proof the infinitesimal generator of $\left\{C_{t, 1}: t>0\right\}$ is denoted by $A$.

From the proof of Lemma 1 in [12] we know that, given $r \in \mathbb{N}$, there exists a constant $C_{1}=C(r)$ such that, for each $f \in X$ and $t>0$, there is $g_{t} \in D\left(A^{r+1}\right)$ satisfying

$$
\begin{aligned}
& \left\|f-g_{t}\right\|_{X} \leq \sup _{0<h \leq t}\left\|\left(I-C_{h, 1}\right)^{r} f\right\|_{X}, \\
& \left\|A^{r+1}\left(g_{t}\right)\right\|_{X} \leq C_{1} \frac{1}{t^{r+1}} \sup _{0<h \leq t}\left\|\left(I-C_{h, 1}\right)^{r} f\right\|_{X}
\end{aligned}
$$

and

$$
\left\|(-A)^{r}\left(g_{t}\right)\right\|_{X} \leq C_{1} \frac{1}{t^{r}} \sup _{0<h \leq t}\left\|\left(I-C_{h, 1}\right)^{r} f\right\|_{X} .
$$

As in [9], for $\lambda>0$ and $f \in X$, consider the best approximation

$$
E_{\lambda}(f)=\inf \left\{\|f-P\|_{X}: P \in \mathbb{P}_{\xi(\lambda)}\right\} .
$$

It was proved there (Theorem 6.1) that there exists a constant $C_{2}=C(r, \alpha, \beta)$ such that, for $\lambda>0$ and $f \in X$,

$$
E_{\lambda}(f) \leq C_{2} K_{r+1}\left(f, \lambda^{-r-1}\right),
$$

and (Theorem 3.2) for each $Q \in \mathbb{P}_{\xi(\lambda)}$,

$$
\left\|\Psi^{r}(Q)\right\|_{X} \leq C_{2} \lambda^{r}\|Q\|_{X}
$$

Now, fix $\lambda>0$ and $P \in \mathbb{P}_{\xi(\lambda)}$. Let $g_{t} \in D\left(A^{r+1}\right)=\Psi^{r+1}(X)$ (see (18)) be given as (23)-(25) with $t=1 / \lambda$ and $f=P$.

For $\varepsilon>0$ and $k \in \mathbb{N}_{0}$, choose

$$
q\left(g_{t}, k\right) \in \mathbb{P}_{\xi\left(2^{k} \lambda\right)}
$$

such that

$$
\left.\| g_{t}-q\left(g_{t}, k\right)\right) \|_{X} \leq(1+\varepsilon) E_{2^{k} \lambda}\left(g_{t}\right) .
$$

From (26), (18), and (24) we know that

$$
\begin{aligned}
\left.\| g_{t}-q\left(g_{t}, k\right)\right) \|_{X} & \leq C_{2}(1+\varepsilon) K_{r+1}\left(g_{t},\left(2^{k} \lambda\right)^{-r-1}\right) \\
& \leq \frac{C_{2}(1+\varepsilon)}{\left(2^{k} \lambda\right)^{r+1}}\left\|\Psi^{r+1}\left(g_{t}\right)\right\|_{X} \\
& =\frac{C_{2}(1+\varepsilon)}{\left(2^{k} \lambda\right)^{r+1}}\left\|A^{r+1}\left(g_{t}\right)\right\|_{X}
\end{aligned}
$$




$$
\begin{aligned}
& \leq \frac{C_{1} C_{2}(1+\varepsilon)}{\left(2^{k} \lambda\right)^{r+1}} \frac{1}{t^{r+1}} \sup _{0<h \leq t}\left\|\left(I-C_{h, 1}\right)^{r} P\right\|_{X} \\
& =\frac{C_{1} C_{2}(1+\varepsilon)}{\left(2^{k}\right)^{r+1}} \sup _{0<h \leq 1 / \lambda}\left\|\left(I-C_{h, 1}\right)^{r} P\right\|_{X} .
\end{aligned}
$$

On the other hand, from the identity

$$
q\left(g_{t}, 0\right)-g_{t}=\sum_{k=0}^{\infty}\left(q\left(g_{t}, k\right)-q\left(g_{t}, k+1\right)\right),
$$

(28), (27), (29), and (26), one has

$$
\begin{aligned}
\left\|\Psi^{r}\left(q\left(g_{t}, 0\right)-g_{t}\right)\right\|_{X} & \leq \sum_{k=0}^{\infty}\left\|\Psi^{r}\left(q\left(g_{t}, k\right)-q\left(g_{t}, k+1\right)\right)\right\|_{X} \\
& \leq C_{2} \sum_{k=0}^{\infty}\left(2^{k+1} \lambda\right)^{r}\left\|q\left(g_{t}, k\right)-q\left(g_{t}, k+1\right)\right\|_{X} \\
& \leq C_{2} \sum_{k=0}^{\infty}\left(2^{k+1} \lambda\right)^{r}\left(\left\|q\left(g_{t}, k\right)-g_{t}\right\|_{X}+\left\|g_{t}-q\left(g_{t}, k+1\right)\right\|_{X}\right) \\
& \leq 2 C_{1} C_{2}^{2}(1+\varepsilon) \sup _{0<h \leq 1 \lambda}\left\|\left(I-C_{h, 1}\right)^{r} P\right\|_{X} \sum_{k=0}^{\infty}\left(2^{k+1} \lambda\right)^{r} \frac{1}{\left(2^{k}\right)^{r+1}} \\
& =2^{r+1} C_{1} C_{2}^{2}(1+\varepsilon) \lambda^{r} \sup _{0<h \leq 1 \lambda}\left\|\left(I-C_{h, 1}\right)^{r} P\right\|_{X} \sum_{k=0}^{\infty} \frac{1}{2^{k}} \\
& =C_{3}(1+\varepsilon) \lambda^{r} \sup _{0<h \leq 1 / \lambda}\left\|\left(I-C_{h, 1}\right)^{r} P\right\|_{X} .
\end{aligned}
$$

We also need the inequality (see (18) and (25))

$$
\begin{aligned}
\left\|\Psi^{r}\left(g_{t}\right)\right\|_{X} & =\left\|A^{r}\left(g_{t}\right)\right\|_{X} \leq C_{1} \frac{1}{t^{r}} \sup _{0<h \leq t}\left\|\left(I-C_{h, 1}\right)^{r} P\right\|_{X} \\
& =C_{1} \lambda^{r} \sup _{0<h \leq 1 / \lambda}\left\|\left(I-C_{h, 1}\right)^{r} P\right\|_{X} .
\end{aligned}
$$

From the inequalities given above, for $P \in \mathbb{P}_{\xi(\lambda)}$, we obtain

$$
\begin{aligned}
\left\|\Psi^{r}(P)\right\|_{X} & \leq\left\|\Psi^{r}\left(P-q\left(g_{t}, 0\right)\right)\right\|_{X}+\left\|\Psi^{r}\left(q\left(g_{t}, 0\right)\right)\right\|_{X} \\
& \leq C_{2} \lambda^{r}\left\|P-q\left(g_{t}, 0\right)\right\|_{X}+\left\|\Psi^{r}\left(g_{t}\right)\right\|_{X}+\left\|\Psi^{r}\left(g_{t}-q\left(g_{t}, 0\right)\right)\right\|_{X} \\
& \leq C_{1} \lambda^{r}\left(\left\|P-g_{t}\right\|_{X}+\left\|g_{t}-q\left(g_{t}, 0\right)\right\|_{X_{\alpha, \beta}}+C_{4} \lambda^{r} \sup _{0<h \leq 1 / \lambda}\left\|\left(I-C_{h, 1}\right)^{r} P\right\|_{X}\right. \\
& \leq C_{5} \lambda^{r} \sup _{0<h \leq 1 / \lambda}\left\|\left(I-C_{h, 1}\right)^{r} P\right\|_{X} .
\end{aligned}
$$

Remark 4.2 The problem of obtaining a Nikolskii-Stechkin inequality for fractional derivatives is open. 
Funding

The coauthors Adell, J.A. and Quesada, J.M. are partially supported by Research Project MTM2015-67006-P.

\section{Competing interests}

The authors declare that they have no competing interests.

Authors' contributions

The authors read and approved the final manuscript.

\section{Author details}

${ }^{1}$ Departamento de Métodos Estadísticos, Facultad de Ciencias, Universidad de Zaragoza, Zaragoza, Spain. ${ }^{2}$ Facultad de Ciencias Físico-Matemáticas, Benemérita Universidad Autónoma de Puebla, Puebla, México. ${ }^{3}$ Departamento de Matemáticas, Universidad de Jaén, Jaén, Spain.

\section{Publisher's Note}

Springer Nature remains neutral with regard to jurisdictional claims in published maps and institutional affiliations.

Received: 16 February 2018 Accepted: 20 June 2018 Published online: 28 June 2018

\section{References}

1. Askey, R., Wainger, S.: A convolution structure for Jacobi series. Am. J. Math. 91, 463-485 (1969)

2. Bavinck, H.: Jacobi Series and Approximation. Mathematical Center Tracts, vol. 39. Mathematisch Centrum, Amsterdam (1972)

3. Bavinck, H.: On positive convolution operators for Jacobi series. Tohoku Math. J. 24, 55-69 (1972)

4. Bustamante, J.: Some inequalities related to the moduli of smoothness of polynomials. Jaen J. Approx. 3(1), 1-14 (2011)

5. Butzer, P.L., Berens, H.: Semi-Groups of Operators and Approximation. Springer, Berlin (1967)

6. Butzer, P.L., Nessel, R.J.: Fourier Series and Approximation, Vol. I: One-Dimensional Theory. Birkhäuser, Basel (1971)

7. Butzer, P.L., Stens, R.L., Wehrens, M.: Approximation by algebraic convolution integrals. In: Prolla, J. (ed.) Approximation Theory and Functional Analysis, pp. 71-120. North-Holland, Amsterdam (1979)

8. Dai, F., Ditzian, Z.: Littlewood-Paley theory and a sharp Marchaud inequality. Acta Sci. Math. 71, 65-90 (2005)

9. Ditzian, Z:: Fractional derivatives and best approximation. Acta Math. Hung. 83, 323-348 (1998)

10. Ditzian, Z: Estimates of the coefficients of the Jacobi expansion by measures of smoothness. J. Math. Anal. Appl. 384, 303-306 (2011)

11. Nikolskii, S.M.: On linear method of summation of Fourier series. Izv. Akad. Nauk SSSR, Ser. Mat. 12(3), 259-278 (1948)

12. Scherer, K.: Bernstein-type inequalities in a Banach space. Math. Notes 17(6), 555-562 (1975)

13. Stehckin, S.B.: Generalization of some Bernstein inequalities. Dokl. Akad. Nauk SSSR 60(9), 1511-1514 (1948)

14. Szegö, G.: Orthogonal Polynomials, 3rd edn. Amer. Math. Soc. Coll. Publ., vol. 23 (1967)

15. Trebels, W.: Multipliers for $(C, \alpha)$-Bounded Fourier Expansions in Banach Spaces and Approximation Theory. Lecture Notes in Mathematics, vol. 329. Springer, Berlin (1973)

16. Trebels, W.: Equivalence of a K-functional with the approximation behavior of some linear means for abstract Fourier series. Proc. Am. Math. Soc. 127(10), 2883-2887 (1999)

17. Trebels, W., Westphal, U.: Characterizations of K-functionals built from fractional powers of infinitesimal generators of semigroups. Constr. Approx. 19, 355-371 (2003)

18. Wang, Y., Cao, F.: Approximation by semigroups of spherical operators. Front. Math. China 9(2), 387-416 (2014)

19. Westphal, U.: An approach to fractional powers of operators via fractional differences. Proc. Lond. Math. Soc. (3) 29 , 557-576 (1974)

\section{Submit your manuscript to a SpringerOpen ${ }^{\circ}$ journal and benefit from:}

- Convenient online submission

- Rigorous peer review

- Open access: articles freely available online

- High visibility within the field

- Retaining the copyright to your article

Submit your next manuscript at $\boldsymbol{~ s p r i n g e r o p e n . c o m ~}$ 